\title{
Evaluation De L'effet De L'extrait Aqueux Des Feuilles De Abrus Precatorius (Fabaceae) Sur Le Poids Et La Prise Alimentaire Chez Des Rats
}

\section{Marius Lebri, PhD}

Laboratoire de Pharmacodynamie Biochimique, UFR Biosciences,

Université Félix Houphouët Boigny, Abidjan Côte d'Ivoire

Centre de Recherche en Ecologie, Unité de Microbiologie et Biotechnologie,

Université Nangui Abrogoua, Abidjan Côte d'Ivoire

\section{Stéphanie Marianne Lagou, PhD}

UFR Science de la Nature,

Université Nangui Abrogoua Abidjan Côte d'Ivoire

\section{Calixte Bahi, PhD}

Laboratoire de Pharmacodynamie Biochimique, UFR Biosciences,

Université Félix Houphouët Boigny, Abidjan Côte d'Ivoire

\section{Guédé Noel Zirihi, PhD}

Laboratoire de Botanique, UFR Biosciences,

Université Félix Houphouët Boigny, Abidjan, Cote d'Ivoire

\section{Adama Coulibaly, PhD}

Laboratoire de Pharmacodynamie Biochimique, UFR Biosciences,

Université Félix Houphouët Boigny, Abidjan Côte d'Ivoire

\section{Fatia Chigr, PhD}

Mohamed Najimi,

Laboratoire de Génie Biologique, Faculté des Sciences et Technique,

Université Sultan Moulay Slimane Béni-Mellal, Maroc

\section{Abderrafia Hafid, Mostafa Khouili,}

Laboratoire de Chimie Organique et Analytique, Faculté des Sciences et

Technique, Université Sultan Moulay Slimane Béni-Mellal, Maroc

Doi:10.19044/esj.2020.v16n15p365 URL:http://dx.doi.org/10.19044/esj.2020.v16n15p365

\section{Resume}

Abrus precatorius est reconnue dans le monde pour ses nombreuses vertus thérapeutiques en médicine traditionnelle. Des études ont montré que l'extrait aqueux de ses feuilles est non toxique par voie orale chez des rats, ce qui pourrait justifier son innocuité observée chez l'homme en médicine traditionnelle. La présente étude a pour objectif de rechercher l'effet de 
l'extrait aqueux des feuilles de A. precatorius sur le poids corporel et la prise alimentaire des rats traités contre les témoins traités à l'eau distillée. Au cours de cette étude expérimentale de 21 jours, les animaux au nombre de 12 ont été répartis dans des cages individuelles puis regroupés en deux groupes de 6 . Ils ont été gavés chaque jour à deux doses $(2 \mathrm{ml} / 100 \mathrm{~g})$ des substances d'essai (matin/soir) pendant 7 jours et observés pendant 14 jours après l'arrêt du gavage. Afin de déterminer l'influence de l'extrait à la dose efficace (40 $\mathrm{mg} / \mathrm{ml}$ ) sur la croissance et la prise alimentaire des rats, l'évolution de ces deux paramètres a été suivie périodiquement tout au long de l'expérimentation. Les resultats obtenues montrent que l'extrait aqueux des feuilles de A. precatorius à la dose efficace $(40 \mathrm{mg} / \mathrm{ml})$ ne provoque pas de changement sur le poids et l'alimentation des rats. Tous ces résultats viennent justifier les nombreuses utilisations de la plante Abrus precatorius en médicine traditionnelle.

Mots clés: Abrus Precatorius, Médicine Traditionnelle, Poids, Prise Alimentaire, Rats 


\title{
Assessment of the Effect of the Abrus Precatorius's (Fabaceae) Leaves Aqueous Extract on Weight and Food Intake in Rats
}

\section{Marius Lebri, PhD}

Laboratoire de Pharmacodynamie Biochimique, UFR Biosciences, Université Félix Houphouët Boigny, Abidjan Côte d'Ivoire Centre de Recherche en Ecologie, Unité de Microbiologie et Biotechnologie, Université Nangui Abrogoua, Abidjan Côte d'Ivoire

\section{Stéphanie Marianne Lagou, PhD}

UFR Science de la Nature,

Université Nangui Abrogoua Abidjan Côte d'Ivoire

\section{Calixte Bahi, PhD}

Laboratoire de Pharmacodynamie Biochimique, UFR Biosciences,

Université Félix Houphouët Boigny, Abidjan Côte d'Ivoire

\section{Guédé Noel Zirihi, PhD}

Laboratoire de Botanique, UFR Biosciences,

Université Félix Houphouët Boigny, Abidjan, Cote d'Ivoire

\section{Adama Coulibaly, PhD}

Laboratoire de Pharmacodynamie Biochimique, UFR Biosciences,

Université Félix Houphouët Boigny, Abidjan Côte d'Ivoire

\section{Fatia Chigr, PhD}

Mohamed Najimi,

Laboratoire de Génie Biologique, Faculté des Sciences et Technique,

Université Sultan Moulay Slimane Béni-Mellal, Maroc

\section{Abderrafia Hafid, Mostafa Khouili,}

Laboratoire de Chimie Organique et Analytique, Faculté des Sciences et

Technique, Université Sultan Moulay Slimane Béni-Mellal, Maroc

\begin{abstract}
Abrus precatorius is recognized worldwide for its many therapeutic virtues in traditional medicine. Studies have shown that the aqueous extract of its leaves is nontoxic orally in rats, which could justify its harmlessness observed in humans in traditional medicine. The purpose of this study is to investigate the effect of aqueous extract of $\mathrm{A}$. precatorius leaves on body
\end{abstract}


weight and feed intake of the treated rats against distilled water controls. During this 21-day experimental study, the 12 animals were distributed in individual cages and then grouped into two groups of six. They were force-fed every day with two doses $(2 \mathrm{ml} / 100 \mathrm{~g})$ of the test substances. (Morning / evening) for 7 days and observe for 14 days after stopping force-feeding. In order to determine the influence of the extract at the effective dose $(40 \mathrm{mg} /$ $\mathrm{ml}$ ) on the growth and feeding of the rats, the evolution of these two parameters was periodically monitored throughout the experiment. The results obtained show that the aqueous extract of $A$. precatorius leaves at the effective dose $(40 \mathrm{mg} / \mathrm{ml})$ does not cause any change in the weight and diet of the rats. All these results justify the many uses of the plant Abrus precatorius in traditional medicine.

Keywords: Abrus Precatorius, Traditional Medicine, Weight, Food Intake, Rats

\section{Introduction}

Abrus precatorius Lin est une plante grimpante de la famille des Fabaceae, elle est probablement la plus connue du genre Abrus Adans qui contient à peu près 17 espèces trouvées en Afrique, en Madagascar, en Indochine (Schrire, 2005; Prenner, 2013, Lébri et al., 2015). Les feuilles à goût sucré sont très prisées en Madagascar et au Sénégal dans le traitement de la toux, surtout la toux infantile. Ainsi, Abrus precatorius est classée parmi les plantes antitussives (Gairola et al., 2010). Le décocté des feuilles est utilisé au Nigeria dans le traitement du diabète (Ezuruike et Prieto, 2014). A Madagascar, les feuilles servent aussi dans le traitement des irritations du col de la vessie et les graines sont utilisées pour lutter contre les ophtalmies, les ulcères, le lupus, et le cancer (Anonyme, 2010).

En Côte d'Ivoire, une enquête ethnobotanique a montré que les feuilles de Abrus precatorius sont utilisées au Sud du pays, chez les peuples Abbey et Krobou d'Agboville, dans une boisson pour faciliter l'accouchement des femmes (N'guéssan et al., 2010). Vue les nombreuses utilisations des feuilles de Abrus precatorius en médicine traditionnelle, des études toxicologiques ont été entreprises et ont révélé que la plante est non toxique par voie orale chez des rats (Lébri et al., 2015 et 2016) ce qui pourrait justifier son innocuité observée chez l'homme en médicine traditionnelle. L'étude de la bioactivité des feuilles de Abrus precatorius a montré que la plante est riche en groupe chimique naturelles (alcaloïdes, tanins, flavones, coumarines, saponines, stérols, triterpènes et des composés réducteurs), possède un effet anticancer in vitro sur une lignée de cellule tumorale mastocytome P815 (Lébri et al., 2015) et un effet potentiel antidiabétique (Lébri et al., 2019) avec une bonne régulation de la glycémie à la dose efficace 
(40 mg/ml).Tous ces travaux effectués sur la plante, pourraient justifier ses effets avérés en médicine traditionnelle dans le traitement du cancer et du diabète. La présente étude consistait à évaluer l'effet de l'extrait aqueux des feuilles de Abrus precatorius sur le poids et la prise alimentaire chez des rats traités par voie orale contre un lot témoin afin d'apprécier l'influence de cet extrait sur la croissance et l'alimentation des animaux au cours du traitement.

\section{I- Matériel et méthodes \\ I-1- Matériel végétal}

Les feuilles de Abrus precatorius ont été récoltées dans les environs de la ville d'Abidjan en Côte d'Ivoire. La plante été identifiée au Centre National de Floristique (CNF) de l'Université Félix Houphouët Boigny, Cocody, Abidjan sur le numéro : Abrus precatorius (Fabaceae) : AboudéMandéké (Côte d'Ivoire), 23 mai 1990, N'guessan Koffi 165 (N'guéssan et al., 2010). Les feuilles séchées à l'abri du soleil ont été broyées et la poudre obtenue a été utilisée pour la préparation de l'extrait.

\section{I-2- Matériel animal}

Les animaux expérimentaux sont des rats blancs mâles de souche Wistar âgés de 8 à 9 semaines dont le poids moyen est $180 \mathrm{~g}$. Les rats ont été placés dans des cages plastiques contenant des copeaux de bois renouvelés tous les 3 jours.

Les rats mâles ont été acclimatés aux conditions de laboratoire (une température de 20 à $22{ }^{\circ} \mathrm{C}, 12$ heures de lumière et 12 heures d'obscurité) pendant 7 jours. Les animaux au nombre de 12 ont été repartis dans des cages individuelles puis regroupés en deux lots homogènes de 6 . Ils ont été mis à jeun pendant 18 heures avant l'administration de l'extrait par gavage. Les animaux ont été privés de nourriture mais pas d'eau, ils sont issus de l'animalerie de la Faculté des Sciences et Techniques de BeniMellal, Université Sultane Moulay Slimane (Maroc).

\section{2- Préparation de l'extrait aqueux des feuilles de Abrus precatorius}

L'extrait total aqueux des feuilles de Abrus precatorius a été préparé en suivant la méthode préconisée en médecine traditionnelle (décoction) (Konkon et al., 2006) modifiée par Lébri et al., 2015.

Dix (10) g de poudre de la feuille de Abrus precatorius ont été repris dans $100 \mathrm{~mL}$ d'une solution d'eau distillée dans un ballon à tricône de $250 \mathrm{~mL}$ surmontée d'un réfrigérant connecté à un robinet ouvert à l'aide d'un tuyau. Le tricône surmonté du réfrigérant est disposé dans un chauffe-ballon maintenu à une température $\left(50^{\circ} \mathrm{C}\right)$ de chauffage constante pendant une heure. Après refroidissement, le mélange a été filtré 3 fois sur du coton hydrophile et mise à l'étuve à $55^{\circ} \mathrm{C}$ pendant $24 \mathrm{~h}$. Le séchage a permis d'obtenir une poudre 
sèche de couleur marron (ETAAP). L'opération a été reprise plusieurs fois pour obtenir une quantité d'extrait suffisante pour la réalisation des tests.

\section{3. Effet de l'extrait aqueux des feuilles de Abrus precatorius sur le poids et la prise alimentaire chez les rats}

L'extrait aqueux des feuilles de Abrus precatorius à la dose efficace (40 mg/ml pour $2 \mathrm{ml} / 100 \mathrm{~g}$ p.c) a été utilisé pour les différentes expérimentations.

Au cours de cette étude de 21 jours, les animaux au nombre de 12 ont été repartis dans des cages individuelles puis regroupés en deux groupes de 6. Ils ont été gavés chaque jour à deux doses $(2 \mathrm{ml} / 100 \mathrm{~g})$ des substances d'essai (matin/soir) pendant 7 jours :

Groupe 1: témoin recevant de l'eau distillée

Groupe 2: recevant 1'ETAAP à la dose efficace $(40 \mathrm{mg} / \mathrm{ml})$

Au premier jour ( 0 ) avant le traitement le poids de base et la prise alimentaire des animaux a été effectué. L'évolution du poids et de la prise alimentaire des rats ont été suivies toutes les semaines après le premier jour (J0) pendant le gavage de 7 jours et toutes les semaines après l'arrêt du gavage jusqu'au $21^{\text {ème }}$ jour.

\section{3.1 Effet de l'extrait sur l'évolution du poids corporel des rats}

L'évolution du poids corporel des rats traités et témoins a été suivie périodiquement tout au long de l'expérimentation. Le poids corporel est mesuré en gramme (g), à l'aide d'une balance électronique (SARTORIUS) et les variations du poids corporel des rats par rapport au $1^{\mathrm{er}}$ jour sont exprimées en pourcentage $(\%)$ et calculées selon la formule suivante :$$
\left(\mathbf{P}_{\mathbf{J}}-\mathbf{P}_{\mathbf{J} 0}\right) \times 100
$$

\section{Variation du poids corporel $(\%)=$}

$\mathrm{P}_{\mathrm{J} 0}$ : poids corporel au $1^{\mathrm{er}}$ jour ;

\section{J0}

$\mathrm{P}_{\mathrm{J}}$ : poids corporel au jour $\mathrm{J}$.

\section{3.2 Effet de l'extrait sur la prise alimentaire chez des rats}

Elle a consisté à déterminer la quantité d'aliments consommée en 24 heures par les animaux par rapport à la quantité de nourriture de départ. La méthode a été adaptée de celle décrite par Kask et al.,(1998); Kokare et al., (2006 ); Kotagale et al., (2014). Sur la base que les rats adultes mangent entre 12-30 g d'aliment sec par jour (Anonyme, 2015), $30 \mathrm{~g}$ des granulés ont été déposés dans les cages individuelles des animaux chaque jour pendant la période d'étude. Avant le gavage selon les lots d'essai, la prise alimentaire a été suivie (J0). La prise alimentaire journalière a été calculée selon la formule suivante : 
Prise alimentaire $(\mathrm{g})=\mathbf{N t}-(\mathbf{N i}+\mathbf{N g})$

$\mathbf{N t}$ : quantité de nourriture totale $(\mathrm{g})$

Ni: quantité de nourriture intacte après alimentation des rats

$\mathbf{N g}$ : quantité de nourriture gaspillée (résidus) après alimentation

\section{I- 4 Analyse statistique}

Le logiciel est Graph Pad Prism (version 5.01, Software, USA) et les résultats sont donnés sous forme de moyennes \pm écart-types. L'évaluation statistique est effectuée en utilisant l'analyse des variables (Anova one way) tests de comparaisons multiples de Dunnett et test de Student pour la comparaison entre deux groupes.

\section{II- Résultats et discussion}

\section{II- 1 Évolution du poids à moyen terme}

Une augmentation non-significative $(P>0,05)$ du poids corporel des rats témoins est enregistrée, à une semaine du gavage. Le poids passe de 191,2 $\pm 11,74$ à $197,3 \pm 13,02 \mathrm{~g}$ soit une augmentation de $\mathbf{3 , 1 9} \%$.

Une semaine après l'arrêt du gavage des rats (J14), l'augmentation du poids enregistrée est non significative de l'ordre de $\mathbf{1 , 7 2} \%$ par rapport au premier jour. Au $21^{\text {ème }}$ jour, le poids des rats augmente de manière non-significative de l'ordre de $\mathbf{4 , 9 1 \%}$, il passe à 200,6 $\pm 26,99 \mathrm{~g}$. Ces résultats montrent une augmentation non significative du poids corporel des témoins au cours de la période expérimentale d'étude (figure 1a).

Chez les rats gavés à deux doses de l'extrait (ETAAP $40 \mathrm{mg} / \mathrm{ml}$ ), une diminution non significative $(\mathrm{P}>0,05)$ du poids corporel a été enregistrée à une semaine du gavage, le poids passe de 192,9 \pm 7,53 à 192,0 $\pm 11,47 \mathrm{~g}$ soit une diminution de $\mathbf{0 , 4 7} \%$.

Une semaine après l'arrêt du gavage le poids diminue de manière non significative, il passe à 191,3 $\pm 14,01 \mathrm{~g}$ soit une diminution de $\mathbf{0 , 3 6} \%$. Au $21^{\text {ème }}$ jour, le poids passe à 191,0 $\pm 18,63 \mathrm{~g}$ soit une diminution non significative de $\mathbf{0 , 9 8} \%$. Ces résultats montrent une diminution non significative du poids corporel des rats traités au cours de la période de 21 jours (figure 1b).

Toutefois, une différence non-significative $(P>0,05)$ de la variation du poids corporel est enregistrée chaque semaine entre les groupes au cours de 21 jours (figure 1c). 


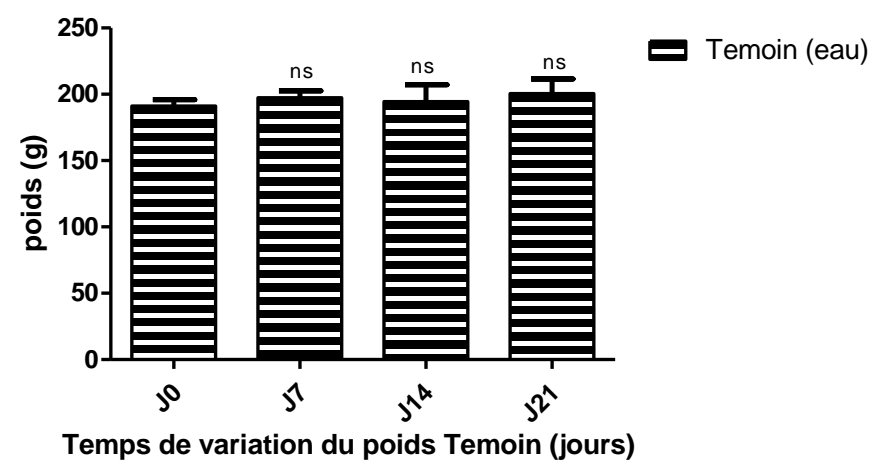

Figure 1a : Variation du poids corporel pendant 21 jours chez les rats normaux soumis à un gavage quotidien d'une semaine à deux doses du véhicule

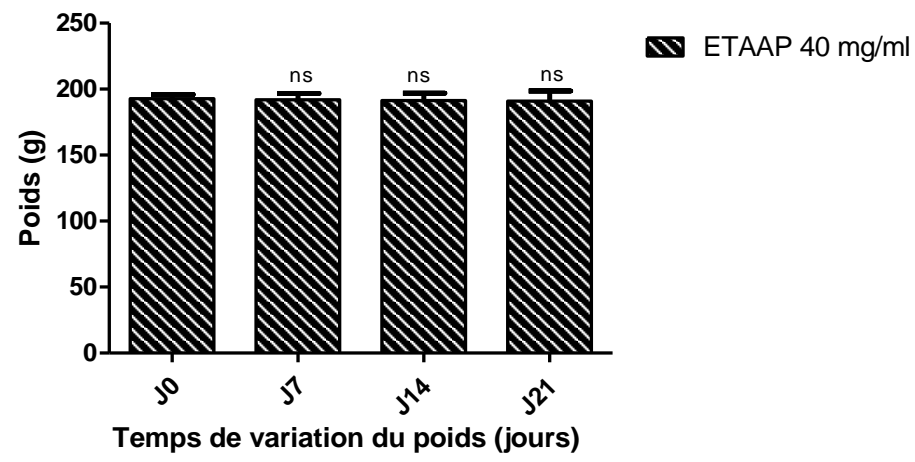

Figure 1b: Variation du poids corporel pendant 21 jours chez les rats normaux soumis à un gavage quotidien d'une semaine à deux doses d'extrait total aqueux des feuilles de Abrus precatorius (ETAAP $40 \mathrm{mg} / \mathrm{ml}$ )

Test de comparaison multiple de Dunnett

ns $P>0,05:$ non significative par rapport au $\mathrm{JO}(n=6)$

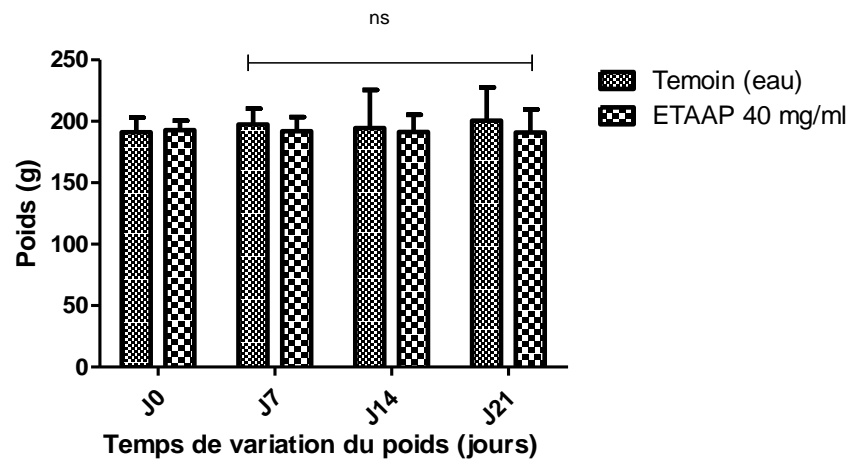

Figure 1c: Variation du poids corporel pendant 21 jours chez les rats normaux soumis à un gavage quotidien d'une semaine à deux doses de l'extrait total aqueux des feuilles de Abrus precatorius (ETAAP $40 \mathrm{mg} / \mathrm{ml}$ ) contre le témoin (eau)

Test de Student : ns P>0,05 


\section{II -2 Evolution de la prise alimentaire à moyen terme}

Dans le lot témoin, à une semaine du gavage des rats, une augmentation non significative $(P>0,05)$ de la prise alimentaire est enregistrée, elle passe de $13,32 \pm 2,29 \mathrm{~g}$ à $17,62 \pm 3,74 \mathrm{~g}$ soit une augmentation de $\mathbf{3 2 , 2 8 \%}$.

Une semaine après l'arrêt du gavage (J14), la prise alimentaire augmente de manière significative $(P<0,01)$, elle passe à $20,98 \pm 3,7 \mathrm{~g}$ soit une augmentation de $\mathbf{5 7 , 5 0 \%}$.

$\mathrm{Au} 21^{\text {ème }}$ jour, une augmentation non significative $(P>0,05)$ de la prise alimentaire de l'ordre de $\mathbf{3 2 , 5 0 \%}$ est enregistrée, elle passe à 17,65 $\pm 3,61 \mathrm{~g}$.

Ces résultats montrent que la prise alimentaire chez les rats témoins augmente de manière non significative à une semaine $(\mathrm{J} 7)$ du gavage et elle devient significative une semaine (J14) après l'arrêt du gavage au cours de la période de 21 jours (figure 2a).

Chez les animaux gavés avec l'extrait (ETAAP 40), à une semaine une augmentation non significative $(P>0,05)$ de la prise alimentaire a été observée à (J7). Elle passe de $13,17 \pm 4,79$ à 17,92 $\pm 4,141 \mathrm{~g}$ soit une augmentation de $36,06 \%$.

Une semaine après l'arrêt du gavage (J14), la prise alimentaire des rats augmente de manière non significative, elle passe à $14,57 \pm 4,59 \mathrm{~g}$ soit une augmentation de $10,63 \%$.

$\mathrm{Au} 21^{\text {ème }}$ jour, la prise alimentaire enregistrée augmente de manière non significative, passe à $17,07 \pm 2,841 \mathrm{~g}$ soit une augmentation de $\mathbf{2 2 , 8 4 \%}$ par rapport au premier (J0).

Les résultats obtenus ne montrent pas d'augmentation significative de la prise alimentaire au cours de 21 jours, après une semaine de gavage des rats normaux à l'extrait (ETAAP $40 \mathrm{mg} / \mathrm{ml}$ ) (figure 2a).

Les résultats des figures $\mathbf{2 a}$ et $\mathbf{b}$ ont montré une augmentation non significative $(P>0,05)$ de la prise alimentaire à une semaine du gavage dans les deux groupes. Au $14^{\text {ème }}$ jour soit une semaine après l'arrêt du gavage, l'augmentation de la prise alimentaire est significative $(P<0,01)$, chez les rats témoins tandis qu'elle devient non significative chez les traités.

Toutefois, une différence non significative $(\mathrm{P}>0,05)$ de la variation de la prise alimentaire est enregistrée entre les deux groupes au cours de la période de 21 jours (figure2c). 


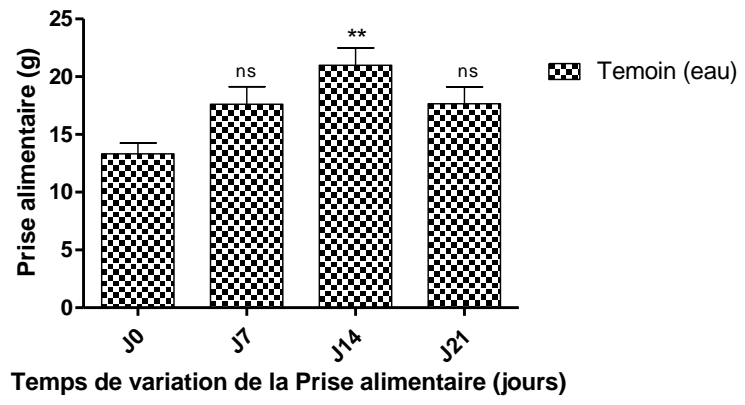

Figure 2a : Variation de la prise alimentaire pendant 21 jours chez les rats normaux soumis à un gavage quotidien d'une semaine à deux doses du véhicule (eau)

Test de comparaisons multiples de Dunnett $n s P>0,05:$ non significative par rapport au $\mathrm{JO}(n=6)$

$* * P<0,01:$ signification par rapport au JO

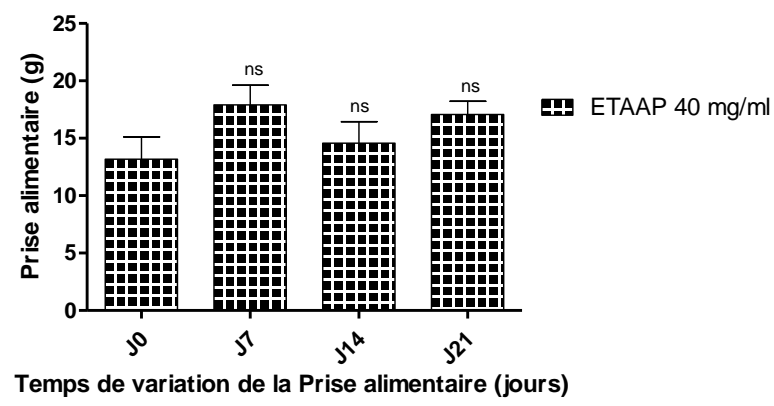

Figure 2b: Variation de la prise alimentaire pendant 21 jours chez les rats normaux soumis à un gavage quotidien d'une semaine à deux doses de l'extrait total aqueux des feuilles de Abrus precatorius à $40 \mathrm{mg} / \mathrm{ml}$

Test de comparaisons multiples de Dunnett $n s P>0,05:$ non significative par rapport au $\mathrm{JO}(n=6)$

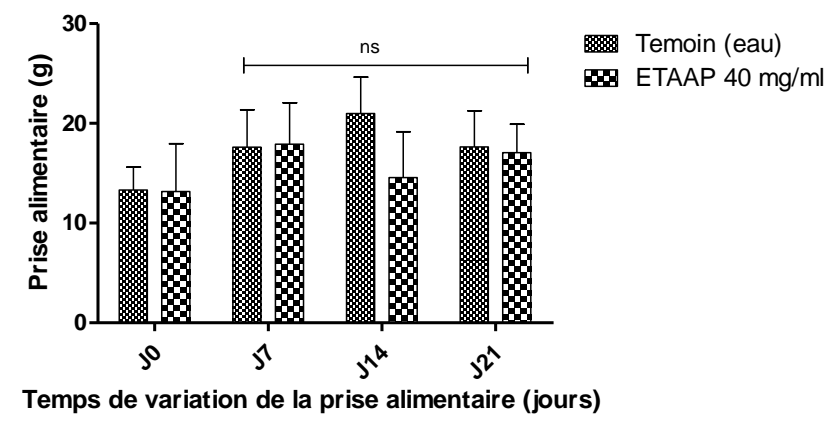

Figure 22c : Variation de la prise alimentaire pendant 21 jours chez les rats normaux soumis à un gavage quotidien d'une semaine à deux doses de l'extrait total aqueux des feuilles de Abrus precatorius à $40 \mathrm{mg} / \mathrm{ml}$ contre le témoin (eau)

Test de Student : ns P>0,05 


\section{III- Discussion}

Les résultats de l'évolution du poids et de la prise alimentaire ont montré une différence non significative de ces paramètres entre les deux groupes. L'extrait aqueux des feuilles de Abrus precatorius à $40 \mathrm{mg} / \mathrm{ml} \mathrm{ne}$ provoque pas de variation significative sur l'évolution du poids. L'extrait aqueux des feuilles de A precatorius ne contiendrait pas de substances capables d'influencer l'évolution du poids. Ce résultat est proche de celui de Koné et al., 2009 dont les travaux ont montré que l'extrait aqueux des écorces de Sacoglottis gabonensis (Baille) Urban (Humiriaceae) à toutes les doses ne provoque pas de variation significative du poids corporel chez les rongeurs. Aucune variation significative n'a été observée sur la prise alimentaire des rats après une semaine de traitement à l'extrait aqueux des feuilles de Abrus precatorius $(40 \mathrm{mg} / \mathrm{ml})$. L'extrait n'a pas eu d'influence sur le comportement alimentaire des rats, il ne contiendrait pas de substances capables de réduire la consommation de nourriture des rats durant le traitement. Ce résultat est contraire à celui de Kotagale et al., 2014, dont les travaux ont montré que la pentazocine par un effet anorexigène, réduit la consommation de nourriture chez les rats en fonction de la dose.

\section{Conclusion}

La présente étude à montré que l'extrait aqueux des feuilles de Abrus precatorius ne provoque pas de changement important sur la variation du poids et de la prise alimentaire chez les rats. Ces resultats pourraient justifier les nombreuses utilisations de la plante en médicine traditionnelle pour son innocuité constatée lors des traitements. Cependant, des études doivent être entreprises à long terme pour mieux apprécier l'effet de la plante sur l'évolution du poids et de la prise alimentaire durant et après les traitements.

\section{Remerciements}

Les auteurs expriment leur gratitude au Laboratoire de Pharmacodynamie Biochimique, UFR Biosciences, Université Félix Houphouët Boigny, Abidjan (Côte d'Ivoire) et à l'Université Sultan Moulay Slimane de Beni Mellal, Faculté des sciences et techniques du Maroc (Maroc) pour avoir fourni les installations nécessaires à la conduite de cette étude.

L'auteur principal tient à remercier le Centre de Recherche en Ecologie de l'Université Nangui Abrogoua Côte d'Ivoire pour l'assistance institutionnelle.

\section{References:}

1. Abboud J., Aoun J., Jammal B., El Rammouz R. \& Saab A.M. (2015). Effect of chemical accumulation and temperature on fish production in Lebanese water ponds. International Journal of Fisheries and Aquatic Studies, 3(1): 43-47. 
2. Schrire B. ( 2005). Tribe Abr eae. In: Lewis, G., Schrire, B., Mackinder, B., Lock, M. (Eds.), Legumes of the World. Royal Botanic Gardens, Kew

3. Prenner, G. (2013). Flower development in Abrus precatorius (Leguminosae: Papilionoideae: Abreae) and a review of androecial characters in Papilionoideae. South African Journal of Botany, 89: 210-211

4. Lébri M., Bahi C., Fofié Y.B.N., Gnahoué G., Lagou S.M., Achibat H., Yapi A., Zirihi G.N., Coulibaly A., Hafid A., Khouili M. (2015) Analyse phytochimique et évaluation de la toxicité aiguë par voie orale chez des rats de l'extrait total aqueux des feuilles de Abrus precatorius Linn (Fabaceae). International Journal of Biological and Chemical Sciences, 9, 1470-1476

5. Garaniya N, Bapodra A. (2014). Ethno botanical and Phytophrmacological potential of Abrus precatorius L. Asian Pacific Journal Tropical Medecin, 4: S27-S34

6. Ezuruike U.F., Prieto J.M. (2014). The use of plants in the traditional management of diabetes in Nigeria: Pharmacological and toxicological considerations. Journal of Ethnopharmacology , 155(2): 859-860.

7. Anonyme (2010). Association Jardin du Monde. Abrus precatorius (MM) (consulté le Lundi, 26 Avril 2010 16:07). Disponible à l'adresse

8. www.jardinsdumonde.org/index.php?...article...abrus-precatorius.

9. N'Guessan K, Zirihi N.G., Boraud N.K.M. (2010). Etude ethnopharmacologique des plantes utilisées pour faciliter l'accouchement, en pays Abbey et Krobou, au Sud de la Côte-d'Ivoire. Internal Journal of Biological Sciences 4: 1009-1010

10. Marius Lébri, Stéphanie Marianne Lagou, N’guéssan Bra Yvette Fofie, Calixte Bahi, Guédé Noel Zirihi, Adama Coulibaly, Fatiha Chigr, Mohamed Najimi, Abderrafia Hafid

11. and Mostafa Khouili. (2019). Criblage phytochimique et effet antidiabétique de l'extrait aqueux de Abrus precatorius Linn (Fabacea). Science et technique, Sciences de la santé, Vol. 42, $\mathrm{n}^{\circ} 2$ : ISSN 1011- 6028

12. Konkon N.G., Simaga D., Adjoungova A.L., N 'Guessan K.E., Zirihi G.N., Kone B.D. (2006). Etude Phytochimique Demitragyna Inermis (Willd.) O. Ktze (Rubiaceae),

13. plante à feuille antidiabetique. Pharmacopée et médecine traditionnelle africaine 15: 73-74.

14. Anonyme (2015). Chapitre XXI. Les rats d'expérimentation, Manuel du CCPA sur le soin et l'utilisation des animaux d'expérimentation, vol. 2(1984) 
15. Kask, A., Rago, L., Harro, J. (1998). Evidence for involvement of neuropeptide Y receptors in the regulation of food intake: studies with Y1-selective antagonist BIBP3226. British Journal of Pharmacology,124: 1507-1515.

16. Kokare, D.M., Patole, A.M., Carta, A., Chopde, C.T., Subhedar, N.K., (2006). GABA (A)

17. receptors mediate orexin-A induced stimulation of food intake. Neuropharmacology 50, 16-24.

18. Koné M, Bleyere NM, Yapo A. P., Vangah M. O., Ehilé E. E. (2009). Evaluation de la toxicité d'un extrait aqueux de Sacoglottis gabonensis (Baille) Urban (Humiriaceae) chez les rongeurs, une plante utilisée dans le traitement de l'ulcère de Buruli en Côte d'Ivoire. International Journal of Biological and Chemical Sciences, 3(6): 1286-1296

19. Kotagale N.R , Upadhya M. , Hadole P.N. , Kokare D.M. , Taksande B.G. (2014)

20. Involvement of hypothalamic neuropeptide $Y$ in pentazocine induced suppression of food intake in rats. Neuropeptides. 48 (3): 133-41.

21. Anonyme (2015). Chapitre XXI. Les rats d'expérimentation, Manuel du CCPA sur le soin et l'utilisation des animaux d'expérimentation, vol. 2(1984) 